\title{
Simplified estimation of membrane potentials induced by high-frequency electric signals
}

\author{
Mehrdad Saviz ${ }^{1}$ and Reza Faraji-Dana ${ }^{2}$ \\ 1. School of Electrical and Computer Engineering, University of Tehran, Tehran, Iran. \\ 2. Center of Excellence on Applied Electromagnetic Systems, University of Tehran, Tehran, Iran. \\ 3. E-mail any correspondence to: msaviz@ut.ac.ir.
}

\begin{abstract}
In this paper we show why the poorly conducting cytoplasmic membranes have little effect on the overall impedance of the tissue above a certain frequency, and derive an estimate of this upper frequency. It is further shown that the induced transmembrane potentials at different sites over the membrane can be found through a simple formula for frequencies above the threshold, without the need to analytically or theoretically model the membranes directly. The findings are validated for an irregular cell shape through rigorous numerical modeling.
\end{abstract}

Keywords: Cytoplasm Membranes, Numerical Modeling, Transmembrane Potential, High Frequencies.

\section{Introduction}

There has been a general research trend towards exploring possible health hazards arising from wireless communication systems, and with it an increasing interest in electrical modeling of tissues from knowledge of sub-tissue and cellular details and exploring possible effects [1]. Schwan et al. [2,3], were among the first to provide a framework for understanding the electrical behavior of tissues as an assembly of cells, and to employ a spherical cell model and the solution of Laplace's equation to arrive at an analytical model for the transmembrane potential (TMP) induced through the external field across the membrane model. Theoretical estimation of bio-impedance and induced membrane voltages are useful in studying electroporation in membranes, which might be applicable for treatment of certain cancers [4].

While analytical formulas might provide good TMP models for simple cell shapes, they fail to provide the accurate TMP distribution for more complex cell shapes. The need to provide estimates for more realistic cell shapes have been shown [5-7], and numerical electromagnetic modeling has been employed together with several cell models [8-11]. In these works, however, in order to evaluate the induced TMP, the membranes are often included in the computational mesh directly. Obviously, this increases the computational complexity because the numerical grid (mesh) has objects with extremely large contrasts in size: the membrane model on the order of several nanometers, and the inner volume mesh-cells on the order of micrometers. With most electromagnetic simulation engines, such an arrangement can lead to increased computational load, and poor quality results [12]. To remedy this problem at very low frequencies, it has been shown that the membranes can be substituted by a zerothickness boundary condition [12]. Upon numerical simulation, the new model will produce the same field results in all regions, and a voltage drop at the interface equal to that of the original model. This method is applicable to low frequencies, however.

This paper shows why the presence of membranes loses significance in the overall impedance, and can be neglected in theoretical estimations of tissue impedance after a certain frequency, and further shows how to calculate the induced TMP without directly modeling the membranes at such frequencies. This obviously reduces the number of mesh elements, allows uniform element sizes for better matrixconditioning, and makes computer simulation of multiplecell tissue models possible.

\section{Materials and methods}

In order to investigate the effect of thin cell membranes on the impedance at sufficiently high frequencies, we first assume fairly general conditions:

(1) The membranes can be considered as insulating, and

(2) They occupy a sufficiently small volume fraction.

We will then find conditions under which the absence of membranes does not affect the impedance and fields obtained at non-membrane regions in the model.

Intuitively, at low frequencies, charging and discharging can occur at the site of poorly conducting membranes in response to the externally induced electric fields, which act more or less as barriers for the free electrolyte charges in the cytoplasm and the extra-cellular aqueous medium. Since the membranes are extremely thin (about $5.5 \mathrm{~nm}$ ), this capacitive charging results in extremely high concentrations of electric field within the membrane, known as the membrane amplification effect [5]. At higher frequencies, where displacement currents dominate over conduction currents, this effect gradually vanishes. Above the transition frequency, the presence of cell membranes in the electrical model has almost no effect on the overall electric field distribution and impedance. 
To investigate this assumption more rigorously, let us consider the local model around the membranes [5,14] as presented in Figure 1. The local field distribution in this region is quasi-electrostatic and can be solved by defining a voltage excitation $V$ in a parallel-plate (capacitor) structure.

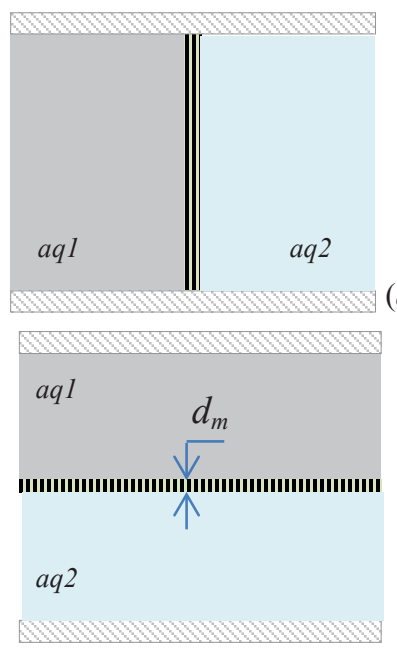

(a)

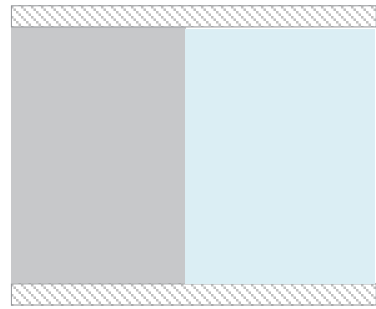

(b)

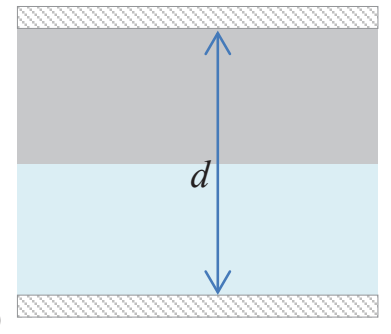

Fig.1: Models for field distribution with and without membranes: (a) The case where the electric field is perpendicular to the membrane; (b) The case where the electric field is parallel to the membrane.

We assume all materials to be non-magnetic, i.e. $\mu=1$ is assumed for all regions. We assume $d=d_{m}+d_{a q}$ and $d_{m} \ll d_{a q}$, which correspond to the case of cytoplasmic membranes being extremely thin and having sufficient volumes of aqueous phase on either side. For all numerical examples below, $d_{m}=5 \mathrm{~nm}$ [5] and $d=100 d_{m}$. It is also worthy of note, that the distributed electrical description for the cell is used here to facilitate discussion, and the physical conclusions obtained are rather general (also valid for mesh-based computation of the fields).

We now compare the impedance of the models with and without membranes in Figure 1 for both polarizations. For fields parallel to the membrane, corresponding to the local models in Figure 1a, the fields in non-membrane regions are obviously independent of the presence of membrane. This is justified by analogy to circuit concepts; parallel capacitors have the same potential, independent of each other and equal to that of the common terminal. For field polarizations perpendicular to the membrane, the series model in Figure 1b can be considered. For the fields in non-membrane regions to be independent of membrane presence, the total current density in models with and without membranes should be the same under equal voltage excitations, which means that the two models should have the same impedance, or that the admittance ratio $M$ in (Eq.1) becomes equal to unity;

$$
M=\frac{d_{a q}+d_{m}\left(\frac{\varepsilon_{a q}}{\varepsilon_{m}}\right)}{d_{a q}+d_{m}} \cong 1+f_{m}\left(\frac{\varepsilon_{a q}}{\varepsilon_{m}}\right)
$$

where $f_{m}=d_{m} /\left(d_{a q}+d_{m}\right)$ is the volume fraction of the membrane in the local model, and $\varepsilon_{a q}=2\left(1 / \varepsilon_{a q 1}+1 / \varepsilon_{a q 2}\right)^{-1}$.

For sufficiently low frequencies (below the first relaxation of pure water around $20 \mathrm{GHz}$ ), we assume the aqueous phase to be described by $\varepsilon_{a q}=\varepsilon_{r}+\sigma / j \omega \varepsilon_{0}$. Inserting this in (Eq.1), we see that for $\omega \ll \sigma / \varepsilon_{r} \varepsilon_{0}$ the admittance ratio can grow unboundedly, while for $\gg$ $\sigma / \varepsilon_{r} \varepsilon_{0}, M \cong 1+f_{m}\left(\varepsilon_{r} / \varepsilon_{m}\right)$. A typical biological solution can be exemplified by $\varepsilon_{r}=60$ and $\sigma=1 \mathrm{~S} \cdot \mathrm{m}^{-1}$ and these values have also been used to model internal cell properties [14], and the membrane can be modeled by a real permittivity of $\varepsilon_{m}=9$ [15]. This yields $|M-1|<0.02$ after a transition frequency of about $f_{T}=100 \mathrm{MHz}$, implying that the same field at non-membrane regions, and the same local admittance is obtained either with or without membranes above $f_{T}$.

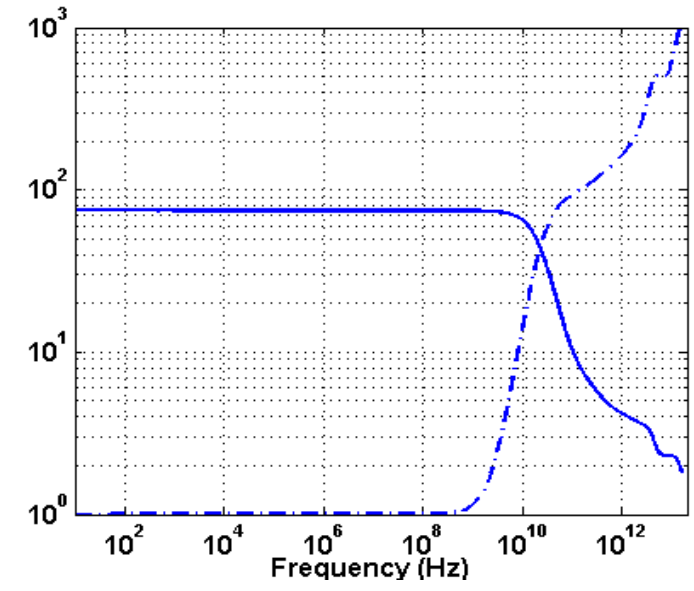

Fig.2: The electrical properties of the aqueous phase: solid line: real permittivity, dash-dotted line: effective conductivity.

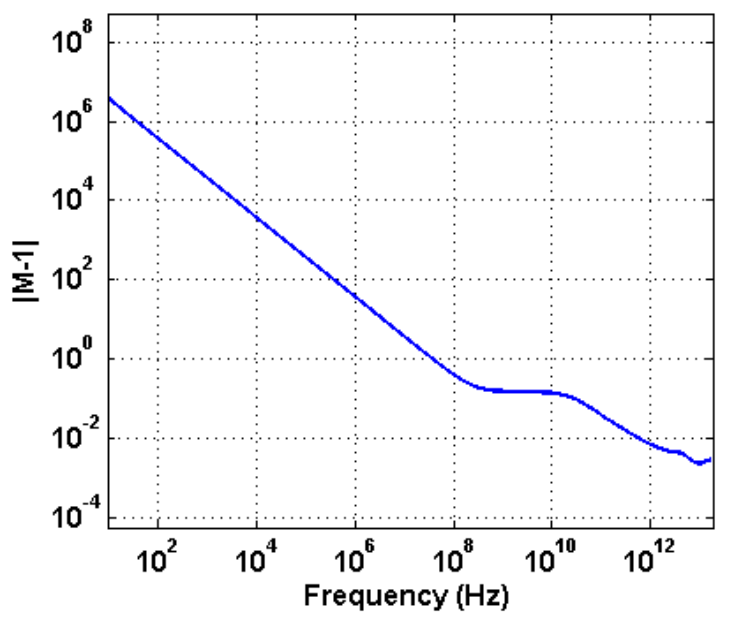

Fig.3: Log plot for the difference of the admittance ratio $\mathrm{M}$ from unity, which shows that approximately from $\mathrm{f}=100 \mathrm{MHz}$ onwards, the presence of membrane becomes insignificant in the overall admittance. 


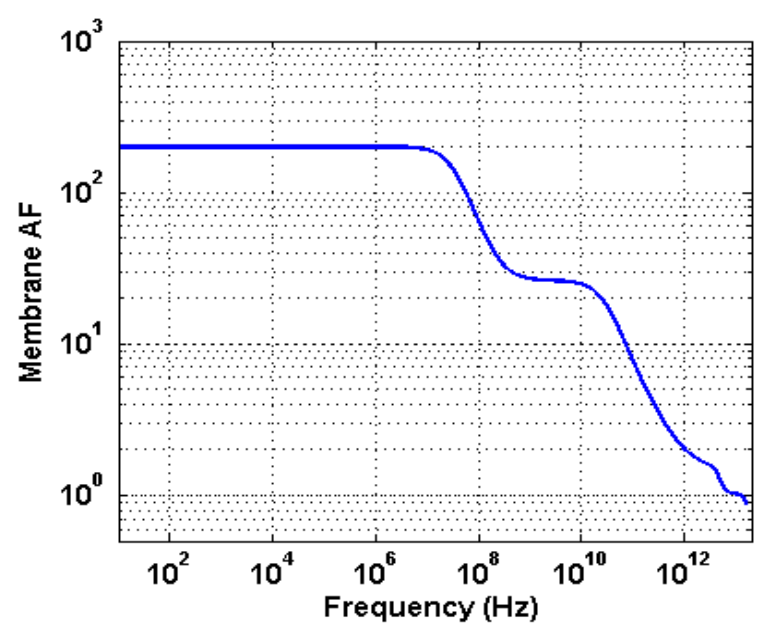

Fig.4: Membrane amplification factor G. The first drop occurs when displacement currents become dominant. The second drop occurs due to water relaxation around $20 \mathrm{GHz}$.

Intuitively, the displacement current dominates in the total current density for $\omega \gg \sigma / \varepsilon_{r} \varepsilon_{0}$, and the conduction current (and the blocking effect of the membrane for free charges) loses significance in determining the overall field. At the same time, even though the conduction current in the aqueous phases gradually increases (due to the increasing field penetration into the cytoplasm, upper-bounded by an average field value in the tissue), the accumulated charge on either side of the membrane unboundedly decreases with frequency, since $\rho_{s}=-J_{c} / j \omega$. This in turn decreases the induced membrane voltage, which is also evident in the corresponding relation for the membrane amplification $G=E_{m} / E,(E$ is the average field strength; $V=E d)$ which for the model in Figure 1b simplifies to (Eq.2).

$$
G=\frac{d_{a q}}{d_{a q}\left(\frac{\varepsilon_{m}}{\varepsilon_{a q}}\right)+d_{m}} .
$$

For $\omega \ll \sigma / \varepsilon_{r} \varepsilon_{0}, G \cong d_{a q} / d_{m}$, which can be quite large considering the extremely thin membrane. For $\omega \gg \sigma /$ $\varepsilon_{r} \varepsilon_{0}, G \cong \varepsilon_{r} / \varepsilon_{m}$, which does not exceed a value of 40 , based on permittivity values from literature $[14,15]$. Consequently, the amplification factor drops to a lower plateau at the same transition frequency as the admittance ratio.

The constant real-part model used for $\varepsilon_{a q}$ does not hold near and above the first water dispersion frequency. To provide a full-spectrum analysis, the complex permittivity of the aqueous phase $\varepsilon_{a q}$ has to be replaced by the frequency-dependent permittivity model for the biological solution. We use a recently published model for pure water which covers frequencies up to $25 \mathrm{THz}[16]$, at $37^{\circ} \mathrm{C}$ and with an additional ionic conductivity term of $\sigma=1 \mathrm{~S} \cdot \mathrm{m}^{-1}$. For this wide-band model, the membrane permittivity is considered to be $\varepsilon_{m}=2.5$, in agreement with the highfrequency values for lipids [17]. The model is graphically shown in Figure 2. The frequency-dependence of $M$ and $G$ are given in Figure 3 and Figure 4. In this more accurate analysis, the transition frequency is seen to be around 100 $\mathrm{MHz}$, in accordance with the previous discussion. The amplification factor undergoes a second decrease as the permittivity of water decreases drastically after $20 \mathrm{GHz}$. Remarkably, the admittance ratio remains near unity, and thereby supports our procedure for removing the membranes in the numerical model at some higher frequencies as well.

As a side note, we see that heating and thermal absorption of energy from the applied field (proportional to $\left.|\vec{E}|^{2}\right)$ can also increase within the cytoplasm at higher frequencies, as membrane amplification drops and penetration of the field into the cytoplasm increases. This is further justified considering the monotonic increase of cytoplasmic effective conductivity with frequency (Fig. 2, see also [5]).

\section{Retrieving the transmembrane potential}

Having obtained the correct field distributions at all regions (except the membrane) using analytical or theoretical methods, one can obtain the externally-induced transmembrane voltages as a post-processing step by considering (Eq.3) from Maxwell's equations:

$$
\vec{\nabla} \cdot\left[\left(\sigma_{i}+j \omega \varepsilon_{i}\right) \vec{E}_{i}\right]=0
$$

The expression inside the brackets represents the addition of the conduction and displacement currents, which we denote by $\vec{J}_{t}$. It can be shown from (Eq.3) that across any surface, the normal component of $\vec{J}_{t}$ is continuous. It can therefore be concluded that:

$$
J_{t 1}^{\perp}=J_{t m}^{\perp}=J_{t 2}^{\perp}
$$

from which the electric field across the membrane $E_{m}^{\perp}$ can be deduced as in (Eq.5);

$$
\left(\sigma_{m}+j \omega \varepsilon_{m}\right) E_{m}^{\perp}=\left(\sigma_{1}+j \omega \varepsilon_{1}\right) E_{1}^{\perp}
$$

The induced transmembrane voltage due to the external field; $\Delta V_{m}^{\text {ext }}=E_{m}^{\perp} \cdot d_{m}$, expressed in terms of the more useful parameters of membrane capacitance and conductance per unit area, is simply obtained as:

$$
\Delta V_{m}^{e x t}=\frac{\left(\sigma_{1}+j \omega \varepsilon_{1}\right) E_{1}^{\perp}}{\left(g_{m}+j \omega c_{m}\right)}
$$

which can be used to recover the membrane potentials (or electric fields) after obtaining the numerical field results. 


\section{Results}

To verify the proposed procedure, we can compare a direct computation of TMP with the one obtained by removing the membranes from the numerical mesh and using (Eq.6). We can do this numerical experiment for a non-trivial example cell shape.

To elaborate, we use a biconcave cell model with a diameter of $16 \mu \mathrm{m}$, whose least thickness at the center is 4 $\mu \mathrm{m}$. The interior and exterior are filled with the same aqueous medium $\varepsilon_{a q}$. Numerical field simulations have been performed using a commercial electromagnetics solver $\left(\mathrm{CST}-\mathrm{EMS}^{\circledR}\right)$. The normal component of the electric field (transmembrane voltage divided by membrane thickness $d_{m}$ ) is obtained in two ways: first numerically by including the membrane in exact field simulations, and subsequently by removing it from the numerical model and using the field distribution obtained without membranes in (Eq.6). We note that for this example, the removal of membranes always leads to a uniform field (potential distribution) in all regions. The results of both approaches are compared in Figure 5 at frequencies of $1 \mathrm{MHz}, 1 \mathrm{GHz}$, and $500 \mathrm{GHz}$ respectively for an ambient electric field strength of $E=1 \mathrm{~V} / \mathrm{m}$.

\section{Discussion}

At $1 \mathrm{MHz}$, we are far below the characteristic frequency of $f \approx 100 \mathrm{MHz}$, conduction currents dominate, and the membranes cannot be removed from the model. This is observed in Figure 5a, where the actual membrane field does not agree with the use of (Eq.6). The potential distribution around the cell model is also given, which indicates the pronounced effect of the membrane on the otherwise uniform field distribution in other regions. At 1 $\mathrm{GHz}$, however, the potential distribution with membranes agrees with the uniform potential distribution that is obtained without membranes and (Eq.6) faithfully reproduces the actual membrane fields. The agreement continues at higher frequencies, i.e. at $500 \mathrm{GHz}$, though with reduced amplification. Obviously, the numerical results verify the presented approach.

From a computational point of view, this will obviously reduce the number of elements and, more remarkably, help maintain a uniform mesh size across the model. For tightly packed tissue models composed of membranes separating cytoplasmic regions, such simplification may drastically reduce the computational effort by allowing us to solve an almost homogeneous tissue model. Consequently, larger multiple-cell scenarios can be solved with implications for tissue-level electroporation studies [5].

We shall underline our basic assumption of $d_{m} \ll d_{a q}$, or equivalently $f_{m} \ll 1$, which means that the local volume fraction of lipid membranes should be rather small. This condition is essential for constructing a local model such as that in Figure 1, and thus for the whole conclusion to hold.
This condition usually holds for cell membranes, since cells have dimensions typically extending to and beyond several micrometers. It does not hold for dense intracellular arrangements of membranes or the outer segment of retinal rod cells, which have a stacked arrangement of membranous disks. In such cases, the effective medium approach might be used to simplify the computational model.
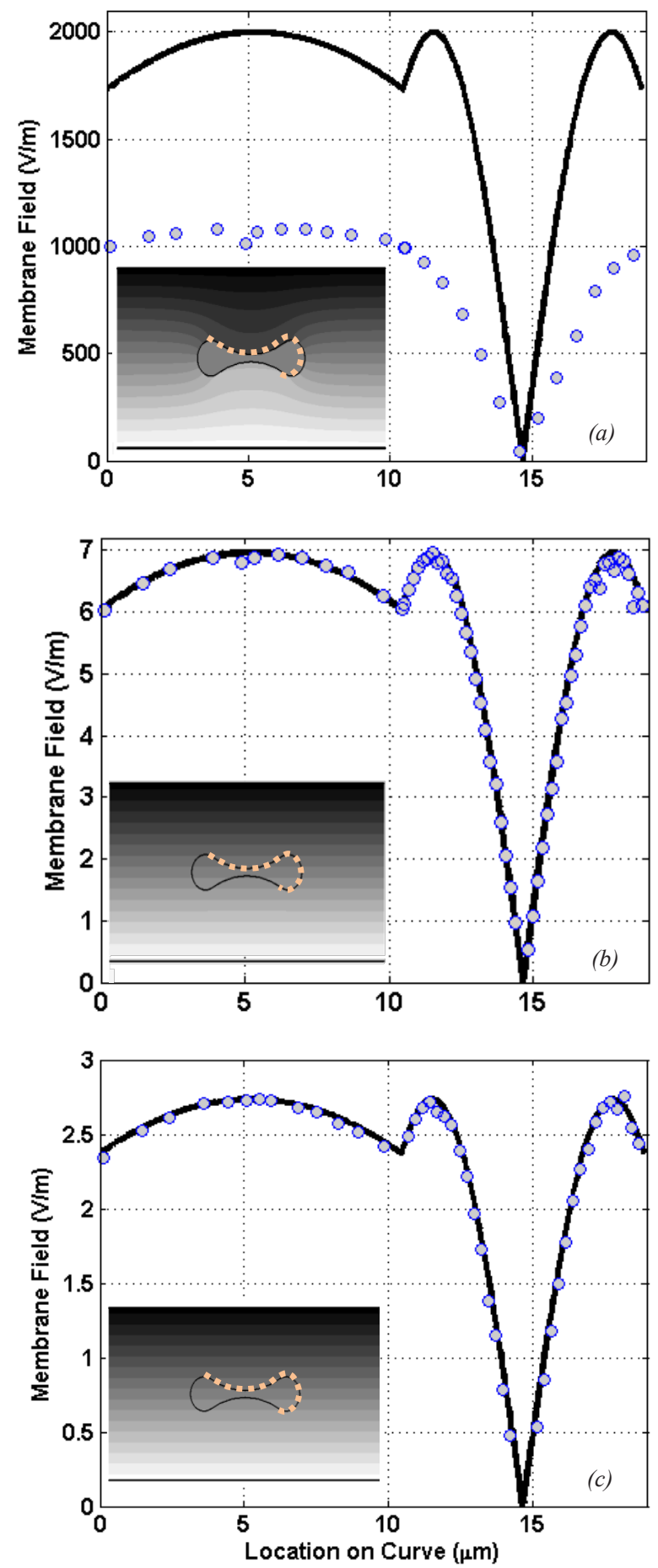

Fig.5: Potential distribution with the presence of membranes, and normal electric field in the membrane (along the dashed curve in the cell membrane); solid line: results of removing membranes in the numerical model and retrieval of membrane fields using (Eq.6), Markers: exact numerical computation of membrane fields, for (a) $1 \mathrm{MHz}$, (b) $1 \mathrm{GHz}$, (c) $500 \mathrm{GHz}$. 


\section{References}

1. Fear EC and Stuchly MA. Modeling Assemblies of Biological Cells Exposed to Electric Fields, IEEE Trans. Biomed. Eng. 1998;45:1259-71. http://dx.doi.org/10.1109/10.720204

2. Grosse $\mathrm{C}$ and Schwan HP. Cellular membrane potentials induced by alternating fields, Biophys. J. 1992;63:1632-42. http://dx.doi.org/10.1016/S0006-3495(92)81740-X

3. Schwan H P and Foster K R. RF-field interactions with biological systems: Electrical properties and biophysical mechanisms. Proceedings of the IEEE 1980;68:104- 113. http://dx.doi.org/10.1109/PROC.1980.11589

4. Schoenbach KH, Nuccitelli R, Beebe SJ. Zap. IEEE Spectrum, 2006;43:20-7.

http://dx.doi.org/10.1109/MSPEC.2006.1665052

5. Gowrishankar TR and Weaver JC. An approach to electrical modeling of single and multiple cells, PNAS 2003;100:3203-8. http://dx.doi.org/10.1073/pnas.0636434100

6. Munoz San Martin M, Sebastian J L, Sancho M and Miranda J M. A study of the electric field distribution in erythrocyte and rod shape cells from direct RF exposure, Phys. Med. Biol. 2003;48: 1649-59. http://dx.doi.org/10.1088/0031-9155/48/11/311

7. Sebastian JL, Munoz S, Sancho M and Miranda JM. Analysis of the influence of the cell geometry, orientation and cell proximity effects on the electric field distribution from direct RF exposure. Phys. Med. Biol. 2003;46:213-25. http://dx.doi.org/10.1088/0031-9155/46/1/315

8. Liberti M, Apollonio F, Merla C and D'Inzeo G. Microdosimetry in the microwave range: a quantitative assessment at single cell level, IEEE Trans. Antennas and Wireless Propag. Lett. 2009;8:865-9 http://dx.doi.org/10.1109/LAWP.2009.2028045

9. Ying W and Henriquez CS. Hybrid Finite Element Method for Describing the Electrical Response of Biological Cells to Applied Fields IEEE Trans. Biomed. Eng., 1998; 54:611-621.
10. Emili G, Schiavoni A, Francavilla M, Roselli L and Sorrentino R. Computation of Electromagnetic Field Inside a Tissue at Mobile Communications Frequencies, IEEE Trans. MTT 2003;51:178-187. http://dx.doi.org/10.1109/TMTT.2002.806899

11. Sebastian JL, Martın SMS, Sancho M and Miranda JM. Modeling the internal field distribution in human erythrocytes exposed to MW radiation, Bioelectrochemistry, 2004;64: 3945. http://dx.doi.org/10.1016/j.bioelechem.2004.02.003

12. Pucihar G, Kotnik T, Valič B and Miklavčič D. Numerical Determination of transmembrane voltage induced on irregularly shaped cells, Annals of Biomedical Engineering, 2006;34:642-652. http://dx.doi.org/10.1007/s10439-005-9076-2

13. Merla C, Liberti M, Apollonio F, Nervi C and D'Inzeo G. Dielectric spectroscopy of blood cells suspensions: study on geometrical structure of biological cells, Proc Conf. IEEE Eng Med Biol Soc. 2006;1:3194-7.

14. Joshi RP, Hu Q and Schoenbach KH. Modeling Studies of Cell Response to Ultrashort, High-Intensity Electric FieldsImplications for Intracellular Manipulation, IEEE Trans. Plasma Sci., 2004;132:1677-1686.

15. Gimsa J, Müller T, Schnelle T and Fuhr G. Dielectric Spectroscopy of Single Human Erythrocytes at Physiological Ionic Strength: Dispersion of the Cytoplasm Biophys. J., 1996;71:495-506.

16. Ellison WJ. Permittivity of pure water, at standard atmospheric pressure, over the frequency range $0-25 \mathrm{THz}$ and the temperature range $0-100{ }^{\circ} \mathrm{C}$, J. Phys. Chem. Ref. Data 2007;36:2-18. http://dx.doi.org/10.1063/1.2360986

17. Reid C B, Pickwell-MacPherson E, Laufer JG, Gibson AP, Hebden JC, and Wallace V. Accuracy and resolution of THz reflection spectroscopy for medical imaging, Phys. Med. Biol. 2010;55: 4825-38. http://dx.doi.org/10.1088/0031-9155/55/16/013 